\title{
Tragédie et question primordiale : le modèle de la suasoire dans l'Iphigénie de Racine
}

\author{
Jérôme Lecompte \\ Paris-III Sorbonne nouvelle \\ lecomptejerome@gmail.com
}

Il suffit de consulter sa mémoire pour penser l'interrogation comme l'une des constantes génériques les mieux marquées de la tragédie. Dans une recherche d'expressivité dont elle est un maillon essentiel, elle apparaît sous toutes ses formes sémantiques et rhétoriques, de l'interrogation pragmatique - comme suspension de la certitude - à l'interrogation rhétorique, employée pour sa double valeur expressive et argumentative. Les premiers vers de l'Iphigénie de Racine témoignent d'un ébranlement de la vraisemblance logique qui doit paraître de mauvais augure. En effet, l'ordre commun est alors sur le point de s'effondrer tout à fait pour laisser place à un nouveau régime logique, celui du merveilleux, de l'événement imprévisible par essence. L'étonnement d'Arcas est rendu par trois interrogations, qui sont les premiers ressorts poétiques de l'exposition :

\footnotetext{
AgAMEMNON

Oui, c'est Agamemnon, c'est ton Roi qui t'éveille.

Viens, reconnais la voix qui frappe ton oreille.$$
\text { ARCAS }
$$

C'est vous-même, Seigneur ! Quel important besoin

Vous a fait devancer l'Aurore de si loin?

A peine un faible jour vous éclaire et me guide.

Vos yeux seuls et les miens sont ouverts dans l'Aulide.

Avez-vous dans les airs entendu quelque bruit?

Les Vents nous auraient-ils exaucés cette nuit?

Mais tout dort, et l'Armée, et les Vents, et Neptune.
}

$$
\text { (I, 1, v. 1-9) }
$$

La première interrogation permet d'ouvrir un bref état de conjecture portant sur l'éventualité de l'événement indispensable à la traversée de la mer Égée. Elle entraîne à sa suite deux questions orientées qui, sémantiquement chargées des vraisemblances les plus élémentaires, témoignent de l'espoir et de l'inquiétude des Grecs. Mais Arcas comprend vite que ce lever matinal n'est pas le signe de l'événement tant espéré ; les possibles les plus attendus se referment et renforcent l'interrogation première, toujours en suspens. Quelque chose est advenu. Il y a donc bien changement de régime, mais s'il n'appartient pas au domestique d'en deviner à lui seul la nature, c'est que la perturbation s'écarte trop de l'ordre commun. Et de fait, la décision de sacrifier Iphigénie est imprévisible car elle n'est pas immédiatement vraisemblable; elle demande à être justifiée. Pour cela même, cette résolution suscite au cours de la pièce l'incrédulité des différents protagonistes. Comme le laissait pressentir la surprise d'Arcas, l'ordre du monde intelligible s'est fracturé, ouvrant l'abîme de la question, et il faudra formuler maintes raisons vraisemblables pour tenter de le refermer et de restaurer ou refonder cet ordre. Ainsi la mère et la fille ont-elles également pour première réaction de laisser échapper des interrogations où se conjuguent valeur pragmatique et valeur rhétorique :

\author{
Clytemnestre \\ Les Dieux ordonneraient-ils un meurtre abominable? \\ IPHIGENIE \\ Ciel ! pour tant de rigueur de quoi suis-je coupable ?
}

(III, 5, v. 921-922)

Bien que ces interrogations constituent des répliques isolées, elles relèvent d'un dispositif général qui leur vaut le statut de figure macrostructurale. Chacune a pour fonction de souligner le caractère inacceptable de l'oracle, et dénote ainsi la position argumentative que le personnage continuera de tenir ensuite dans la 
scène de confrontation avec Agamemnon (IV, 4). Nulle question ne sera plus formulée alors par Iphigénie, dont la tirade délibérative mettra en avant l'acceptation du sacrifice même de l'innocence. Pour Clytemnestre, en revanche, la qualification infamante du sacrifice comme meurtre (v. 921) interdit de supposer que les dieux se gouvernent en fonction de l'honnête. Associé à leur décision, Agamemnon ne peut donc manquer d'apparaître comme un père dénaturé, parce qu'il n'a pas su ou souhaité leur opposer de résistance :

Quoi ! l'horreur de souscrire à cet ordre inhumain

N'a pas en le traçant arrêté votre main?

Pourquoi feindre à nos yeux une fausse tristesse ?

Pensez-vous par des pleurs prouver votre tendresse?

Où sont-ils ces combats que vous avez rendus?

Quels flots de sang pour elle avez-vous répandus?

Quel débris parle ici de votre résistance?

Quel champ couvert de morts me condamne au silence?

Voilà par quels témoins il fallait me prouver,

Cruel, que votre amour a voulu la sauver.

$$
\text { (IV, 4, v. 1255-1264) }
$$

La fureur de Clytemnestre éclate dans un flot d'interrogations qui réduit Agamemnon au silence, ou, pour mieux dire, le roi est ainsi réduit à quia en se voyant retirer tout moyen de défendre sa position. Répétitive jusque dans la forme, cette expolition insiste sans relâche sur l'absence de preuves suffisantes d'une résistance à l'oracle, et développe ainsi l'opinion que l'attitude d'Agamemnon est hypocrite. Ces premiers exemples permettent de juger que la question et l'interrogation rhétorique suspendent ou renforcent les vraisemblances prises en charge par les différents personnages. Or toutes concernent ici le problème fondamental de la pièce.

Selon un principe rhétorique énoncé par Quintilien, « une question naît d'une question » (Inst. orat., III, $11, \S 3)$, de sorte qu'il en existe une qui précède toutes les autres selon un rapport d'antériorité logique. La traduction littérale de l'adjectif principalis dissimulant trop en français le sème de l'origine derrière celui de l'importance, nous lui avons préféré le terme primordial, qui possède le même champ sémantique. Si l'on reprend maintenant Iphigénie au niveau macrostructural supérieur, c'est la tragédie tout entière dont il est possible de résumer l'argument en ces termes : Agamemnon doit-il sacrifier Iphigénie ? Nous proposons de vérifier pour la pièce l'importance de cette quasstio principalis théorisée par les rhétoriciens de l'antiquité, auxquels nous nous adresserons dans cette étude, parce que la «question primordiale» nous semble non seulement exemplaire de la nature problématique de la rhétorique, mais encore du genre même de la tragédie.

Poser la question primordiale permet de générer des arguments vraisemblables. C'est donc un moteur de l'invention et de la disposition des arguments à l'aune du discours ou de l'enchaînement des scènes. Malgré quoi il ne s'agira pas d'entreprendre ici une génétique poétique, à la manière de Georges Forestier pour Corneille, mais bien plutôt une génétique rhétorique. Nous tenterons de relier l'emploi des formes interrogatives et des assertions vraisemblables à cette « question primordiale » en laquelle nous pourrions voir une source logique et technique de l'invention des arguments. En dernière analyse, nous évaluerons la pertinence d'une étude systématique de la tragédie selon ce modèle.

\section{La question primordiale}

Agamemnon doit-il sacrifier Iphigénie ? Cette question résume l'argument des pièces d'Euripide et de Racine, et tel est précisément l'unique sujet de suasoire mythologique proposé par Sénèque le Rhéteur dans le recueil des Sentences, divisions et couleurs des orateurs et des rhéteurs, publié en 39, et souvent réédité du XV $\mathrm{X}^{\mathrm{e}}$ au XVII ${ }^{\mathrm{e}}$ siècle ${ }^{1}$. Sans donner d'exemple achevé, Sénèque a rassemblé en fagots plusieurs arguments de dissuasion imaginés par des rhéteurs qu'il avait connus; il ne s'agissait pour eux que de faire apprécier leur talent dans l'inventio des sentences et des couleurs (apparences ou prétextes), et dans la dispositio d'arguments dont la force persuasive dépendait de l'enchaînement. Cette circonstance s'est avérée déterminante dans le choix d'Iphigénie. 
Dans les écoles des rhéteurs, sous l'Empire, les élèves pratiquaient les exercices de déclamation appelés controverse et suasoire, qui les formaient respectivement aux genres judiciaire et délibératif. Les sujets proposés étaient fictifs et relevaient des questions définies ou hypothèses, qui faisaient référence à un cas particulier («Caton doit-il se marier?»), par opposition aux questions indéfinies, appelées thèses ( « Le

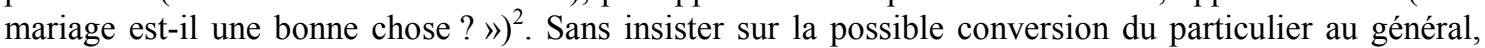
que Cicéron regardait comme un procédé rhétorique nécessaire à l'orateur, il faut remarquer avec Quintilien que l'une et l'autre demandent que l'on puisse apporter « deux ou plusieurs thèses plausibles (in utramque partem vel in plures dici credibiliter)» (Inst. orat., III, XI, § 1) ${ }^{3}$. Les Grecs nomment zêtêma la question primordiale, qui engendre toujours d'autres questions (§§ 3-4). L'élève avait pour tâche de composer un discours fictif prononcé par un personnage historique ou mythologique, pour lequel il fallait inventer les arguments et rechercher les figures les plus appropriées au caractère et aux circonstances, comme dans cet autre exercice rhétorique appelé prosopopée (sermocinatio $)^{4}$. Pour cela, précise Quintilien, on pouvait s'inspirer d'auteurs comme Ménandre, dont les personnages variés et bien campés correspondent parfaitement aux thèmes des controverses $(\mathrm{X}, \mathrm{I}, \S 71)$. La déclamation scolaire n'est pas étrangère à la littérature, et les œuvres de Virgile ou d'Ovide en portent témoignage. Pour un auteur du XVII ${ }^{\mathrm{e}}$ siècle, la formation rhétorique dépend également de ce commerce avec la littérature antique. Il faut insister sur l'importance des passions dans la suasoire comme dans la tragédie, ce qui n'est pas défavorable à leur réunion :

[La suasoire] réclame tout particulièrement l'appel aux émotions, car il y a souvent lieu d'exciter ou de calmer la colère et d'inciter l'esprit à la peur, à la convoitise, à la haine, à la conciliation. Parfois même, il faut éveiller la pitié, soit qu'il faille persuader de porter secours à des assiégés, soit que nous ayons à déplorer la ruine d'une cité alliée.

(Inst. orat., III, 8, § 12)

Enfin, toujours avec Quintilien, il est précieux d'observer que la suasoire exige de la part de l'orateur beaucoup de sagesse et de moralité ( $(13)$. Cette condition est assez bien remplie par Agamemnon et Iphigénie, moins par Ulysse, qui apparaît dans la pièce en qualité de sophiste, et moins encore par Clytemnestre et Achille, trop passionnés pour envisager le problème avec sérénité. C'est là que nous surprenons l'intérêt poétique de la combinaison de la suasoire avec la prosopopée.

A cause de son attrait pour la véritable éloquence, toutefois, l'âge classique marque superbement ses réserves à l'égard de la dimension scolaire de la rhétorique, comme il apparaît chez René Rapin et SaintÉvremond. Ce point nous intéresse, car dans les Réflexions sur l'usage de l'éloquence de 1671, le premier renvoie en ces termes au début du Satiricon :

Il ne faut que consulter l'Agamemnon de Pétrone pour comprendre le ridicule de cette éloquence qui n'a rien de naturel, parce qu'elle s'attache trop aux ornements extérieurs, qu'on veut faire passer pour ce qu'elle a de plus essentiel. Le véritable fonds de l'éloquence ne peut être que le bon sens.

(Réflexions sur l'usage de l'éloquence, I, XV, 34)

Saint-Évremond a restitué ce même passage dans une belle infidèle, qu'il a fait suivre d'un abrégé d'éloquence mondaine, publiant le tout en 1681 sous le titre «Fragment de Pétrone. De l'éloquence ${ }^{5}$. Cet Agamemnon auquel il est fait allusion est le nom emprunté d'un déclamateur qui doit faire une harangue où il s'agit d'« exhorter le Roy Agamemnon à livrer sa fille Iphigénie qui devait être sacrifiée à Diane suivant l'Oracle, afin de faciliter l'expédition de Troie » (48-49). Très vite il apparaît que l'orateurhistrion succombe à l'asianisme. Invité par des auditeurs à donner son sentiment sur le discours, Eumolpe critique sévèrement le goût du fabuleux dans les déclamations : elles sont si éloignées du réel et si guindées qu'elles ne sauraient rien apprendre aux jeunes gens. Mais Agamemnon survient et lui répond. Il ne fait jamais, dit-il, que s'accommoder au goût du siècle. Et si les déclamateurs corrompent ainsi l'éloquence, la faute en revient aux pères de famille, qui devraient choisir une éducation plus sévère. Saint-Évremond applique ensuite le jugement de Pétrone à son temps ; favorable à l'éloquence naturelle, il constate les méfaits de la rhétorique scolaire dans la poésie depuis Ronsard : « d'ordinaire le reste de la pièce n'est rempli que de grands raisonnements hors d'œuvre, de lambeaux décousus, de lieux communs 
cités sans besoin, et sans discrétion » (102). Et de conclure à propos de l'éloquence que « jamais elle ne saurait être trop scrupuleuse à rejeter ce qui peut blesser la vraisemblance » (115) : c'est dire combien la déclamation y répugne par son inclination pour le brillant. Ces réserves exprimées à la fin du XVII ${ }^{\mathrm{e}}$ siècle témoignent de l'actualité du problème ${ }^{6}$. Le choix du naturel aura pour effet de dissimuler l'art, c'est-àdire de soustraire l'artifice au regard du spectateur. En lutte contre la mode naissante des tragédies en musique, Racine montre qu'un sujet de déclamation peut donner lieu à un traitement vraisemblable, tant au niveau poétique (le dénouement évite le merveilleux) qu'au niveau rhétorique (les arguments sont vraisemblables aux différents plans de l'invention, de la disposition, et de l'élocution, puisqu'il évite l'asianisme critiqué par Eumolpe-Saint-Évremond). C'est une fois de plus vers Quintilien que nous nous tournerons pour évoquer le critère commun à la rhétorique et à la tragédie, le vraisemblable logique.

Au cours de l'examen du champ sémantique d'argumentum, Quintilien observe que le terme ne désigne plus seulement le sujet d'une pièce, mais tout sujet de discours (V, X, §§ 9-10). Cette extension rend manifeste le lien entre l'intrigue et la preuve, puisque l'argumentation rhétorique s'appuie nécessairement sur des prémisses plus ou moins probables, vraisemblances dont la caractéristique fondamentale est de ne pas être toujours vraies, mais ordinairement valables (voir II, XVII, § 39). Pour autant, une telle constance n'exclut pas les anomalies, sans quoi les prémisses seraient indubitables et l'entreprise de persuasion céderait la place à une démonstration :

\begin{abstract}
Pour se servir correctement des arguments, il faut connaître la valeur et la nature de chaque chose et ses effets ordinaires : de là naît ce que les Grecs appellent eikota (la vraisemblance). Dans les choses vraisemblables, il y a trois degrés : tout d'abord, le plus assuré, parce que se produisant presque toujours, par exemple: «Les enfants sont aimés de leurs père et mère »; le second, qui penche un peu pour ainsi dire vers l'incertain : "Qui se porte bien aujourd'hui ira jusqu'à demain »; le troisième enfin où l'on se borne à ne pas rejeter absolument une assertion : «Un vol commis dans une maison a été commis par l'occupant ».
\end{abstract}

(Inst. orat., $\mathrm{V}, \mathrm{X}, \S \S 15-16)$

Du plus au moins certain, la vraisemblance possède des degrés de modalité qui opposent des valeurs absolues et relatives. La résolution contre nature d'Agamemnon contrevient donc au premier, le plus élevé d'entre eux, puisque l'amour paternel devrait l'emporter sur le dessein du sacrifice. Chez Racine, l'argument absolument vraisemblable est ainsi problématisé par le jeu des circonstances, pour donner le sujet d'une suasoire qui se confond avec l'argument de la tragédie. Mais selon ce principe, la question primordiale n'apparaît pas en toutes lettres dans la pièce. Reformulée par les personnages, elle ne donne pas lieu à des interrogations ouvertes. Deux occurrences méritent l'attention à ce titre.

A l'acte IV, les interrogations d'Ériphile ont pour fonction poétique de préparer le grand monologue délibératif d'Agamemnon. La rivale d'Iphigénie auprès d'Achille a trop de dépit pour croire que les termes de l'alternative sont à égalité :

Hé quoi! Ne vois-tu pas tout ce qu'on fait pour elle?

On supprime des dieux la sentence mortelle,

Et quoique le bûcher soit déjà préparé,

Le nom de la victime est encore ignoré.

Tout le camp n'en sait rien. Doris, à ce silence

Ne reconnais-tu pas un père qui balance?

Et que fera-t-il donc? Quel courage endurci

Soutiendrait les assauts qu'on lui prépare ici ${ }^{7}$.

Une Mère en fureur, les larmes d'une Fille,

Les cris, le désespoir de toute une famille,

Le sang à ces objets facile à s'ébranler,

Achille menaçant tout prêt à l'accabler.

Non, que dis-je, les dieux l'ont en vain condamnée [...].

(IV, 1, v. 1113-1125) 
Dans ces vers, l'interrogation ne possède d'autre valeur que rhétorique. Elle est d'abord une prise à témoin, la négation équivalant alors à une assertion (v. 1113 et 1118); elle est ensuite une fausse question ouverte (v. 1119) qui condamne aussitôt l'une des issues de l'alternative, comme le soulignent l'interrogation rhétorique (v. 1119-1120) et les phrases nominales suivantes : le seul fait d'être irrésolu prouve que le sacrifice n'aura pas lieu. Eriphile fonde cette conviction sur le vraisemblable absolu, qui place Agamemnon en position de faiblesse (v. 1123). A ce moment de la pièce il ne s'agit plus de savoir si le père va sacrifier sa fille ou y renoncer, mais plutôt d'apprendre comment il se laissera gagner à ce dernier parti. Furieux contre Achille (IV, 7), Agamemnon n'a jamais été aussi près d'oublier sa tendresse pour Iphigénie. Puis le doute l'assaille $(\mathrm{IV}, 8)$ et provoque une délibération sincère, représentée par la figure macrostructurale de la dubitation, telle que l'a définie Pierre Fontanier ${ }^{8}$. Toutefois, le seul fait d'énoncer la question marque déjà un basculement significatif dans l'irrésolution, comme annoncé par Eriphile :

Que vais-je faire?

Puis-je leur prononcer cet ordre sanguinaire?

Cruel! A quel combat faut-il te préparer!

Quel est cet Ennemi que tu leur vas livrer ${ }^{9}$ !

Une Mère m'attend, une Mère intrépide,

Qui défendra son sang contre un Père homicide.

Je verrai mes Soldats, moins barbares que moi,

Respecter dans ses bras la Fille de leur Roi.

Achille nous menace, Achille nous méprise.

Mais ma Fille en est-elle à mes lois moins soumise ?

Ma Fille, de l'Autel cherchant à s'échapper,

Gémit-elle du coup dont je la veux frapper?

Que dis-je ? Que prétend mon sacrilège zèle?

Quels vœux en l'immolant formerai-je sur elle?

Quelques prix glorieux qui me soient proposés,

Quels lauriers me plairont de son sang arrosé ?

Je veux fléchir des Dieux la puissance suprême?

Ah ! Quels dieux me seraient plus cruels que moi-même !

Non, je ne puis. [...]

(IV, 8, v. 1433-1453)

Poser la question après que la résolution a déjà été prise indique déjà que la remise en jeu est biaisée. L'expression même de cette volonté apparaît insoutenable (v. 1444-1445), et entraîne une série d'interrogations rhétoriques qui peignent l'aversion qu'il aurait alors pour lui-même. Agamemnon étant incapable de se résoudre à immoler Iphigénie, la vraisemblance absolue est maintenue. Ainsi la suasoire vaut-elle également comme mise à l'épreuve de la validité épistémologique du vraisemblable, critère fondamental de la rhétorique, mais critère participant de la crise de la discipline ${ }^{10}$. Quelles raisons un père peut-il invoquer pour décider un tel acte ? Pour le poète, l'inventio ou genèse des arguments sur cette matière relève du défi oratoire. En conséquence, il apparaît qu'il faut moins rechercher dans la pièce la transcription littérale de la suasoire, sous la forme de vraies questions, que les arguments pro et contra inventés par le poète pour installer la tension délibérative au cœur de la pièce.

\section{Critique du vraisemblable dans Iphigénie par un contemporain}

Pour apprécier l'importance de l'invention des arguments dans la tragédie classique, il est utile de se reporter aux Remarques sur les Iphigénie de M. Racine et de M. Coras (1675), au long desquelles un auteur anonyme fait reproche au poète d'avoir négligé le vraisemblable ${ }^{11}$. «Tout le monde demeure d'accord, écrit-il, que pour rendre une action digne du Théâtre, il faut la rendre vraisemblable ». Ajoutons ici pour entendre tout à fait ce partisan de Coras que l'action ne doit pas sentir la déclamation mais doit répondre à l'exigence de naturel. Or la résolution d'Agamemnon chez Racine, estime ce critique, est invraisemblable. Faute d'être suffisamment étayée, elle contrevient en effet au vraisemblable absolu : 


\begin{abstract}
Quels motifs assez pressants pour inspirer à ce père le dessein barbare d'immoler une fille qui lui est si chère? Quelles machines assez violentes pour lui arracher ce consentement? La nature nous porte à conserver la vie de nos enfants aux dépens de la nôtre. Le soin de les élever et de les défendre est l'éternelle occupation de tous les hommes. C'est une obligation que le sang et les lois nous imposent avec un empire si absolu, que leur ingratitude ne peut même nous en dispenser, et les pères qui ne se sacrifient pas pour le bien de leur famille, sont condamnés de tout le monde. Cependant Agamemnon passe sur ces obligations ; il foule aux pieds tous les devoirs du sang. Il n'écoute point sa propre tendresse pour faire périr une fille qu'il idolâtre, et qui a pour lui des sentiments d'obéissance et de respect, capables de pénétrer le cœur le plus inhumain. Ce n'est pas l'amour de la patrie qui lui fait prendre cette résolution.
\end{abstract}

(Remarques sur les Iphigénie, 795)

Ainsi la résolution d'Agamemnon aurait-elle dû rencontrer l'obstacle de la nature, ou du moins fallait-il supposer une raison capitale pour le faire consentir au sacrifice. Or les arguments sont estimés un peu faibles, car le Roi des rois ne paraît pas si jaloux de sa gloire que l'ambition apparaisse comme un motif suffisant, et il n'est pas mû davantage par un scrupule religieux constant, puisqu'il veut dans un premier temps désobéir à Diane, avant de se soumettre aux dieux venus à lui en songe, et d'alléguer enfin leur volonté face à Iphigénie et Clytemnestre ${ }^{12}$. Ce qui pourrait n'être qu'un trait de la part du critique anonyme ne laisse pas d'être une attaque sérieuse, car il est bientôt ajouté qu'Agamemnon n'est plus crédible quand il invoque un arrêt divin pour persuader autrui : « ce ne sont que des couleurs » (797), par quoi il faut entendre des raisons de second rang : les arguments du déclamateur ne conviennent pas à la tragédie. Ils sont insuffisants. Nous retrouvons en l'espèce la critique classique de l'inventio du déclamateur, qui permet de valoriser l'exigence de naturel. Pour autant, la technique n'en demeure pas moins valable : il faut simplement savoir, selon le mot de Quintilien, cacher l'art. Ainsi, quand il doit accuser l'Iphigénie de Racine du défaut de vraisemblance, le critique anonyme ne peut manquer de produire lui-même de nouveaux arguments, comme appelés par ce vide qu'il entrevoit ou qu'il recherche dans les vers de son rival. Marqué de façon exemplaire au niveau macrostructural par l'interrogation, le sentiment d'une insuffisance encourage l'invention du vraisemblable. L'auteur anonyme allègue ainsi le témoin supposé de la guerre de Troie : à l'en croire, Dictys de Crète «colore bien mieux » la résolution d'Agamemnon en le rendant coupable d'avoir tué une biche consacrée à Diane (798). Le sacrifice est donc une expiation. Mais il utilise aussi bien ses propres ressources, et donne lieu à un moment d'éloquence :

Quelle apparence que ces Dieux exigent une réparation si grande, sans qu'il ait été commis aucun crime qui attire leur courroux ? Quelle apparence qu'un grand Roi comme Agamemnon, quelque Religieux qu'il puisse être, se détermine à leur immoler une victime de cette conséquence, pour apaiser leur indignation que personne n'a méritée ? Ce sacrifice barbare était plus propre à irriter les Dieux, qu'à les apaiser ${ }^{13}$.

(Remarques sur les Iphigénie, 797)

Le défaut de vraisemblance logique motive la génération d'arguments déjà inventés ou tout à fait inédits, mais dont la vraisemblance est dans les deux cas jugée supérieure. De manière significative, il invite à une démonstration de force oratoire, exemplairement servie par des interrogations rhétoriques qui se déploient pour acculer l'adversaire, comme nous l'avons déjà vu faire à Clytemnestre.

\title{
3 Genèse du vraisemblable : les raisons d'Agamemnon
}

Dans la pièce d'Euripide, «mille raisons » déterminaient Agamemnon à renoncer au sacrifice d'Iphigénie. Chez Racine, cependant, conserver cette hyperbole à valeur d'ellipse, - nulle raison n'étant donnée en toutes lettres par le poète grec, - il ne fallait pas y songer : son roi devait fournir au public les raisons vraisemblables par lesquelles il se disposait à reprendre l'engagement arraché par Ulysse. Nécessité poétique, intérêt du public pour le vraisemblable et plaisir de l'argumentation le commandaient au poète. En rapportant les paroles du sophiste, Agamemnon relève donc avec sagacité les arguments utilisés pour le fléchir:

Ulysse en apparence approuvant mes discours, 
De ce premier torrent laissa passer le cours.

Mais bientôt rappelant sa cruelle industrie,

Il me représenta l'honneur et la Patrie,

Tout ce Peuple, ces Rois à mes ordres soumis,

Et l'Empire d'Asie à la Grèce promis.

De quel front immolant tout l'Etat à ma Fille,

Roi sans gloire, j'irais vieillir dans ma Famille !

Moi-même (je l'avoue avec quelque pudeur)

Charmé de mon pouvoir, et plein de ma grandeur,

Ces noms de Roi des Rois, et de Chef de la Grèce

Chatouillaient de mon cœur l'orgueilleuse faiblesse.

Après une captatio benevolentice doucereuse, adaptée à un auditeur éperdu qu'il faut apaiser, l'orateur accumule les images du devoir politique pour terminer par une vision vraisemblable du sort que connaîtrait Agamemnon s'il renonçait au sacrifice. Exagération du possible réprouvé, cet adynaton culmine avec la diatypose du « Roi sans gloire », une vision fulgurante qui doit détourner le destinataire des considérations naturelles pour mieux lui faire envisager combien il s'exposerait alors à un désaveu universel. En « immolant tout l'Etat» à sa fille, en effet, Agamemnon se rendrait coupable de préférer l'intérêt domestique à l'intérêt public, et transgresserait l'ordre vraisemblable auquel sont assujettis les souverains dignes de leur état.

Cependant les paroles d'Ulysse ont pénétré l'esprit d'Agamemnon pour le remplir de l'image la plus avantageuse de lui-même, sans lui arracher encore la résolution attendue. Ce sont les dieux, au fil des songes, qui mènent la persuasion à son terme en apportant leur caution à un acte inhumain (I, 1, v. 83-88). Mais un père raisonnable ne peut oublier longtemps qu'il est un père sans déroger, de sorte que le « funeste artifice » qui consistait à faire venir Iphigénie en Aulide sur un faux prétexte ne débouche pas sur l'acte résolu : la décision est reprise (acte I, sc. 1), confirmée (sc. 5), assumée (actes II à IV), remise sur la balance (acte IV, sc. 8), et dénoncée (sc. 9 et 10), avant que la jeune fille ne prenne le relais de son père défaillant (acte $\mathrm{V}$, sc. 2 et 3 ). Tendue entre deux résolutions, et martelée par les soubresauts de l'hésitation, cette temporalité suspensive est le temps d'Iphigénie. Elle correspond pleinement à la suspension sémantique de l'interrogation, trait stylistique prégnant dans la pièce.

Aux «mille raisons » d'Euripide, Racine a donc substitué un adynaton articulé sur une opposition entre l'honneur et l'infamie. Développée par un sophiste qui choisit l'utile de préférence à l'honnête (ce que les bienséances commandent à l'homme de bien), la vision du « roi sans gloire » risque de jeter le discrédit sur le critère du vraisemblable, dont la manifestation n'est pas logique mais pathétique. Néanmoins, quand Agamemnon donne les raisons qui lui font refuser le geste auquel il avait consenti d'abord, il en va tout autrement. Nous ne rappellerons pas les premiers arguments, dont la valeur d'exposition permet aussi la gradation argumentative, pour nous intéresser plutôt à la dernière raison, qui motive bien davantage le revirement :

Ma Fille qui s'approche, et court à son trépas,

Qui loin de soupçonner un arrêt si sévère,

Peut-être s'applaudit des bontés de son Père.

Ma Fille... Ce nom seul, dont les droits sont si saints,

Sa jeunesse, mon sang, n'est pas ce que je plains.

Je plains mille vertus, une amour mutuelle,

Sa piété pour moi, ma tendresse pour elle,

Un respect, qu'en son cœur rien ne peut balancer,

Et que j'avais promis de mieux récompenser.

$$
\text { (I, 1, v. 112-120) }
$$

A l'image conditionnelle de l'avenir d'Agamemnon, « roi sans gloire », répond une evidentia fortement actualisée par le présent et en phase avec la réalité. Cette vision de la confiance d'Iphigénie est prolongée par une hypothèse vraisemblable qui en augmente la puissance pathétique, obtenue par la tension entre l'absence de crainte et la menace. Fille d'Agamemnon, Iphigénie est surtout un modèle de vertu dont la piété filiale est choisie entre «mille» pour la représenter au plus juste. Placée dans l'ordre du 
vraisemblable par cette innocence qui la rend conforme à la doxa, elle est ainsi préparée à ne pouvoir soupçonner l'obscur dessein d'un père dont elle croit partager les valeurs. Face à la pureté de sa fille, Agamemnon mesure sa propre faiblesse. L'«horreur» si brièvement évoquée par Euripide est ainsi approfondie à travers l'image pathétique d'une tendresse réciproque, et d'une trahison sans égale. De l'aveu même du critique anonyme de Racine, l'argument de la nature est le meilleur motif pour dissuader Agamemnon d'immoler Iphigénie. Cette vraisemblance absolue est une valeur universellement partagée, à la fois commune aux personnages et au public. Le portrait s'avère si poignant pour Agamemnon luimême, qu'il confirme la reprise d'une décision extorquée par un sophiste secondé par les dieux. Il faut donc supposer qu'un autre sens doit être donné à l'oracle :

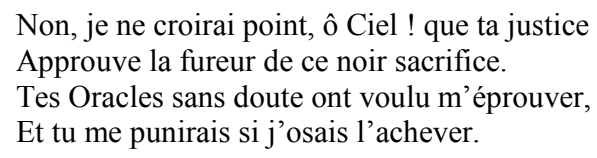

(I, 1, v. 121-124)

La séquence argumentative de la narration est close par une réfutation qui introduit un enthymème dont la logique vraisemblable se laisse reconstruire de la manière suivante : Iphigénie est l'archétype de la jeune fille innocente ; or les dieux seraient injustes s'ils souhaitaient vraiment la voir sacrifier ; une épreuve est donc imposée au roi, les dieux ne pouvant condamner l'innocence : ils châtieraient plutôt un parricide. Cet argument est plus vraisemblable encore pour le public chrétien, pour qui la justice divine est infaillible, et qui reconnaît volontiers dans Agamemnon la préfiguration païenne d'Abraham. Racine achève ainsi le raisonnement par la considération d'un «possible » moralement insoutenable. Dans cette hypothèse finale (v. 124), le dunatôn est l'exact pendant de l'adynaton du « roi sans gloire » qui n'aurait pas sacrifié sa fille, de telle sorte que le récit de la persuasion d'Ulysse et la délibération interne du roi apparaissent bien comme les deux versants contraires et dynamiques de la suasoire, développée ici sous sa première forme. D'autres suivront, qui seront prises en charge par les différents protagonistes, chacun alléguant les raisons que sa position lui désigne comme les plus valables.

A l'issue de cet examen, quelques remarques s'imposent concernant le statut du vraisemblable rhétorique dans la pièce. A cette diversité d'espèce correspond une variété de nature du vraisemblable, les arguments prenant une forme imageante ou abstraite. Dans ce cadre délibératif, les possibles paraissent indifféremment utilisés en sophistique et en rhétorique, par Ulysse et par Agamemnon, mais ils sont alors respectivement ordonnés à l'utile et à l'honnête. Partisan du vir bonus dicendi peritus, Quintilien donnait d'ailleurs la préférence au deuxième critère quand il s'agissait de définir la fin du genre délibératif (III, VIII, § 22). L'enthymème d'Agamemnon développe une logique vraisemblable qui distingue l'orateur du sophiste. Quant à la division, elle est pratiquée à la fois par Ulysse et par Agamemnon, dont les arguments révèlent une hiérarchie interne du vraisemblable. La règle de disposition exigeant de terminer par le plus fort des arguments, les premiers n'ont plus qu'un rôle rhétorique de préparation, auquel s'ajoute un rôle poétique (au sens structural). Mais la hiérarchie des arguments est également externe et absolue, car ils n'ont pas la même valeur : il est plus vraisemblable qu'un souverain néglige les intérêts politiques pour se souvenir qu'il est d'abord un père. Ecarter l'argument de nature n'est somme toute qu'une manœuvre de sophiste pour captiver l'attention en négligeant des circonstances qu'un orateur honnête devrait affronter. Cette faille argumentative sera dénoncée par Agamemnon dans la dernière réplique de la scène 3 :

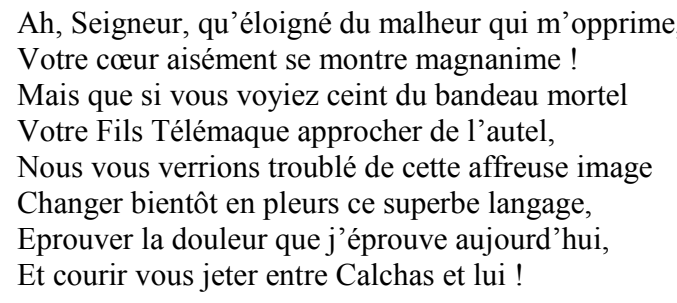

Nouvelle vision projetée où Télémaque à son tour est «ceint du bandeau mortel » réservé aux victimes expiatoires, cet adynaton est une hypothèse impossible qui demeure sans réponse. En effet, alors 
qu'Agamemnon était sur le point de l'emporter, l'irruption d'Eurybate est signe de l'arrivée d'Iphigénie, mais, plus encore, elle est signe que l'argument naturel est véritablement absolu, Racine devant interrompre ici l'entretien par une péripétie. Dans un exorde marqué par la figure de l'homéopathée (harmonie oratoire mais sincère avec l'âme de l'auditeur), Ulysse confirmera le caractère irréfragable de cet argument, que dépasse pourtant la nécessité introduite par l'arrivée d'Iphigénie. Il convient donc de distinguer chez Racine les deux statuts du vraisemblable selon qu'il est utilisé en sophistique ou en rhétorique. In utramque partem, sans doute, c'est-à-dire pro et contra. Pour autant les deux versants contraires de la suasoire ne sont pas mis à égalité ; discours sophistique rapporté d'un côté, délibération sincère de l'autre, la structure rhétorique est ainsi dissimulée pour une meilleure vraisemblance poétique. Nous sommes alors bien loin des «lambeaux décousus» de la suasoire romaine que dénigrait à propos Saint-Évremond. La structure poétique doit permettre de rattraper le défaut de cohérence.

\section{Invention et disposition du vraisemblable délibératif}

Bien que l'incertitude et l'irrésolution d'Agamemnon soient représentées dès la scène d'exposition pour être portées à leur comble dans le monologue délibératif (acte IV, sc. 8), la trame de la suasoire qui soustend la tragédie n'est pas restreinte au personnage principal. D'autres que lui sont les dépositaires d'arguments vraisemblables que le poète distribue in utramque partem, mais en fonction des rôles. La connaissance de l'oracle provoquant une réaction d'horreur unanime, Clytemnestre, Achille et Iphigénie sont naturellement amenés à exprimer le sentiment de leur persona à propos de la résolution d'Agamemnon. La vraisemblance poétique oblige en effet le poète à les faire réagir en conformité avec leur caractère, ce qu'il obtient en leur attribuant les arguments vraisemblables qui leur conviennent. Clytemnestre réagit en mère frappée dans sa chair, le bouillant Achille en amant disposé à venger Iphigénie, trop sûr que le roi n'est plus digne du nom de père. Nous nous arrêterons sur les raisons formulées ensuite par l'hérö̈ne, qui choisit de prendre la défense de son père.

Iphigénie est en effet la dernière à exprimer son sentiment. Les propos d'Achille ont pour elle heurté les bienséances, car elle ne laisse pas de l'appeler son père, quand le héros prétend au contraire qu'il a dérogé ; il faut en conclure que l'acte le plus impie ne suffit pas à briser les liens naturels pour une jeune fille qui incarne la piété filiale ${ }^{14}$, et que la pérennité des lois de la bienséance est ainsi conservée. Dans le bref plaidoyer qu'elle consacre à son père, l'argumentation vraisemblable est développée en deux temps. Iphigénie évoque d'abord une habitude morale devenue consubstantielle par son ancienneté, puis la souffrance nécessaire d'un Agamemnon qui n'en reste pas moins père :

C'est mon Père, Seigneur, je vous le dis encore.

Mais un Père que j'aime, un Père que j'adore,

Qui me chérit lui-même, et dont jusqu'à ce jour

Je n'ai jamais reçu que des marques d'amour.

Mon cœur dans ce respect élevé dès l'enfance,

Ne peut que s'affliger de tout ce qui l'offense.

Et loin d'oser ici par un prompt changement

Approuver la fureur de votre emportement,

Loin que par mes discours je l'attise moi-même,

Croyez qu'il faut aimer autant que je vous aime,

Pour avoir pu souffrir tous les noms odieux,

Dont votre amour le vient outrager à mes yeux.

Et pourquoi voulez-vous qu'inhumain, et barbare,

Il ne gémisse pas du coup qu'on me prépare?

Quel Père de son sang se plaît à se priver ?

Pourquoi me perdrait-il, s'il pouvait me sauver?

J'ai vu, n'en doutez point, ses larmes se répandre.

Faut-il le condamner avant que de l'entendre ?

Hélas! de tant d'horreurs son cœur déjà troublé

Doit-il de votre haine encore être accablé ?

(III, 6, v. 1001-1020) 
La continuité des sentiments rend toute rupture improbable aux yeux d'Iphigénie. Il faudrait apporter des preuves éclatantes du changement moral de son père pour l'en persuader. L'interrogation rhétorique est une fois encore associée à l'expolition (Agamemnon a dû souffrir le premier de cette décision) pour exprimer fortement l'opinion défendue par Iphigénie. Malgré la révélation d'Arcas, malgré la fureur de Clytemnestre et la colère d'Achille, la jeune fille affiche un maintien sublime par la tranquillité de son âme. Or cette stabilité des sentiments se traduit par une fidélité sereine et inattendue envers l'ordre vraisemblable. Tandis que pour une mère et un amant, la résolution d'Agamemnon indique suffisamment que la chaîne logique est brisée, elle n'est pas le signe pour Iphigénie d'un bouleversement complet du cœur de son père. Il lui faut plutôt supposer en toute vraisemblance que des raisons majeures encore inconnues l'ont obligé à ce choix, et qu'il convient d'avance de les respecter.

Massées en fin de réplique, les interrogations rhétoriques mettent en relief les raisons les plus étroitement liées à la question primordiale de la suasoire. Iphigénie reproche à son amant de simplifier le problème à l'excès en dissociant le père et le criminel, de telle sorte qu'il délaisse avec précipitation un ordre logique pour un autre; il ne s'avise pas que sans doute ils sont liés de manière indissoluble, et qu'ils ont jeté Agamemnon dans une douloureuse irrésolution. Un père affectueux ne saurait embrasser le projet de sacrifier sa fille sans frémir. C'est du moins avec cette conviction qu'Iphigénie porte au crédit d'Agamemnon cette loi naturelle, et suppose qu'il a dû considérer toutes les échappatoires possibles avant de conclure à l'inéluctable. Du reste, elle n'appuie pas son raisonnement sur les seules preuves intrinsèques, car elle dispose d'un indice dont le sens, auparavant objet d'erreur, est maintenant certain. Les larmes versées par Agamemnon ne venaient pas du renoncement d'Achille à sa promesse d'épouser Iphigénie, mais d'un oracle trop dur. Il n'est donc pas, comme on le prétend injustement selon Iphigénie, « barbare, injuste, sanguinaire » (III, 6, v. 997), ce n'est donc pas un monstre « du sang d'Atrée et de Thyeste », comme le veut Clytemnestre (IV, 4, v. 1250). Il est homme et se débat dans ses contradictions tout en demeurant fidèle à la nature. C'est par là qu'il justifie que l'on continue de recourir à la logique vraisemblable pour pénétrer les calculs de son esprit. Du moins faut-il préciser que telle est la conviction d'une Iphigénie dont la connaissance du cœur paternel est une importante garantie, que viennent confirmer ensuite le monologue délibératif d'Agamemnon et le revirement qui s'ensuit; la validité épistémologique du vraisemblable est ainsi préservée. Pourtant, malgré une argumentation solide, étayée par une preuve certaine, le plaidoyer d'Iphigénie demeure sans effet sur Achille, dont la plainte amoureuse doit conserver la véhémence caractéristique du héros. C'est le moyen pour Racine de préparer la scène d'altercation avec Agamemnon (IV, 6).

Trois régimes du vraisemblable se succèdent ainsi dans les scènes 5 et 6 de l'acte III, adaptés aux caractères respectifs de Clytemnestre, d'Achille et d'Iphigénie. La mère ne raisonne pas, mais reçoit la résolution d'Agamemnon comme une insulte à la nature, et l'amant se présente comme le vengeur intègre de l'ordre vraisemblable. Mais il revient à Iphigénie de développer les arguments les plus forts, comme son père face à Ulysse, de telle sorte qu'ils sont tous deux les représentants d'un ordre vraisemblable contrarié dont ils possèdent une meilleure connaissance que les autres. Eux seuls ont à l'esprit l'alternative que comporte la question primordiale, eux seuls dépassent l'esprit partisan du pro ou contra. En effet, ni l'un ni l'autre ne font abstraction de la complexité du dilemme, sans laquelle il n'y aurait pas d'irrésolution de la part d'Agamemnon. Cette compréhension intime d'un père tourmenté, accompagnée d'une tranquille assurance qui prépare Iphigénie à consentir au sacrifice, quand elle pourrait enfin y échapper, est un puissant ressort du pathétique. Dès ce moment, la figure d'Iphigénie se détache et commence de s'élever vers le sublime. L'ordonnance poétique de la fable s'appuie sur un rythme rhétorique, celui de la diffusion progressive et mesurée des différents arguments vraisemblables, réductibles au modèle de la suasoire. A travers les deux scènes évoquées, Racine fait dévoiler par ses personnages les positions qu'ils tiendront face à Agamemnon, et à partir desquelles ils tenteront de le dissuader d'immoler Iphigénie. En faisant échapper Iphigénie au sacrifice, Racine parvient donc à concilier deux partis : l'un est épistémologique, et consiste à reconduire la validité de l'ordre du vraisemblable; l'autre est esthétique, et permet l'isolement sublime d'Iphigénie dans la résolution de monter à l'autel pour y être sacrifiée. La perfection de la piété d'Iphigénie est portée à son comble. 
Si la tragédie d'Iphigénie se laisse résumer chez Racine par une question primordiale, le modèle de la suasoire est néanmoins compliqué par la variété des formes du vraisemblable, par la diffusion des arguments adaptés aux caractères et aux intérêts de la dispositio, et, en dernier ressort, par les interactions entre personnages, dans une quête d'exhaustivité qui révèle la contamination de la dramaturgie par le vraisemblable rhétorique. Cette minutieuse inventio engage à ne plus seulement considérer la composition rhétorique de la tragédie en interne, mais dans son lien avec le public. Pour des raisons culturelles et rhétoriques, certains arguments sont attendus, et l'absence de l'un d'entre eux ferait prêter le flanc à la critique. Inventer le vraisemblable demande ainsi de prévoir quelle réfutation pourrait être opposée. Cette forme d'émulation n'est pas sans rappeler la définition de l'inventio fournie par Aristote et Cicéron, qui voulaient que l'orateur recherchât les arguments in utramque partem. Si la logique du vraisemblable paraît confirmée dans la pièce de Racine, elle est toutefois mise en difficulté par le surgissement de circonstances qui n'entrent plus dans l'ordre du monde que connaissaient les personnages, alors conduits à en interroger les contradictions.

Il faut conclure au terme de cette étude à la présence abondante et diversifiée de l'interrogation, qui s'impose exemplairement à tous les niveaux de l'élaboration rhétorique d'Iphigénie. En effet, l'inventio et la dispositio, on vient de le voir, tirent un grand parti de la suasoire, mais l'elocutio l'utilise encore pour traduire la véhémence, et la pronuntiatio s'en empare dans la déclamation; l'éloquence elle-même ne vit que par l'ouverture permanente de ces failles, qu'il faut refermer ou combler au moyen d'arguments vraisemblables. Mais quel bilan tirer des liens entretenus par les deux formes de l'interrogation avec la question primordiale ? Comprise comme modalité de la phrase, l'interrogation permet une suspension transitoire de la vraisemblance, dans une dynamique qui s'exerce sur le double plan rhétorique et poétique. Tel est bien le cas dans le monologue délibératif d'Agamemnon, où la question «Que vais-je faire ? » (IV, 8, 1433), sincèrement posée, introduit la figure macrostructurale de la dubitation. La tirade pose alors sans détour la question primordiale du point de vue d'Agamemnon, tandis que prononcée par Ériphile elle ne pouvait avoir de valeur que poétique, au sens aristotélicien, et encore fixait-elle un horizon d'attente pour le spectateur : «Et que fera-t-il donc ?» (IV, 1, v. 1118), ce roi irrésolu, quand tout le détermine à renoncer au sacrifice, - une mère, une fille, un amant, la nature. Mais il a surtout fallu faire le constat de la présence massive de l'interrogation rhétorique, qui possède une valeur expressive et argumentative de soulignement : les positions vraisemblables sont assumées et même revendiquées par le personnage qui peut rentrer dans l'altercation. Il en va ainsi de la réponse faite par Achille au père d'Iphigénie : « Puis-je entendre et souffrir ce langage ? (IV, 6, v. 1369). La véhémence de la figure est alors renforcée par l'enchaînement de plusieurs interrogations qui interdisent d'autant mieux toute réplique. Néanmoins, dans le cas de cette forme éminemment rhétorique de l'interrogation, le lien génétique avec la question primordiale reste patent: nous l'avons vu précédemment à propos de la réplique d'Iphigénie (v. 1013-1020), mais il apparaît aussi dans le propos d'Achille, qui fait valoir à Agamemnon que rien ne l'engage personnellement contre Troie. Il n'est donc pas de ceux qui avaient intérêt à résoudre le roi au sacrifice de sa fille. En l'espèce, le héros refuse d'être associé malgré lui au parti favorable à cet accomplissement de l'oracle. L'interrogation rhétorique rejoint alors l'assertion vraisemblable, dont elle est une forme intensive.

Pour finir, il reste à évaluer la pertinence du modèle de la suasoire pour une génétique rhétorique de la tragédie. Avec Iphigénie, Racine faisait retour au modèle antique pour répondre au succès grandissant de la tragédie en musique. De là un cas particulier qu'il est difficile de brandir comme exemple type. A tout le moins, Iphigénie présente l'avantage d'accentuer des traits génériques avérés, car il s'agit précisément pour son auteur d'illustrer le genre : la tragédie interroge sans relâche, elle se déploie continûment sur le terrain du probable et le fait in utramque partem, pour ou contre le sacrifice. Nous sommes donc amenés à confirmer la précellence du genre délibératif dans la tragédie classique, telle que Gilles Declercq (2003) l'a établie aux dépens du genre judiciaire en s'appuyant sur une étude de Cinna et d'Iphigénie. Il y observait précisément que Corneille a transformé la question judiciaire tirée de Sénèque (faut-il ou non punir Cinna?) en question délibérative (faut-il régner ou abdiquer ?), c'est-à-dire la controverse en suasoire. Or un bref examen des arguments des tragédies raciniennes permet de confirmer la pertinence du modèle de la suasoire, soit du genre délibératif: Andromaque doit-elle épouser Pyrrhus ? Titus doit-il renoncer à Bérénice? Mithridate doit-il se venger de son fils le plus fidèle ? L'existence au cœur de la 
tragédie d'une «question primordiale » est alors relayée par des interrogations ouvertes, et permet de placer les arguments vraisemblables sous tension. On ne saurait plus parler de « dominante » délibérative, puisque la structure suasoriale impose sa marque à l'argument comme à l'ensemble de la pièce.

Au demeurant, quel avantage par rapport au modèle de la controverse et au genre judiciaire ? On voit qu'il est de nature poétique et esthétique: la problématique délibérative convoque naturellement l'expression des possibles pouvant advenir (figure de la dubitation) et celle des possibles ne devant pas advenir (adynaton), ce qui consiste à privilégier une temporalité suspensive et prospective. Cet aspect n'a d'ailleurs pas échappé au jésuite René Rapin, critique sévère des poètes dramatiques de son époque : dans leurs tragédies, à l'en croire, « on ne met point en usage cette morale, qui est propre à mêler des intérêts différents, des vues opposées, des maximes qui s'entrechoquent, des raisons qui se détruisent les unes les autres, pour fonder ces incertitudes et ces irrésolutions qui font la beauté du théâtre » (Réflexions sur la poétique de ce temps, II, XXI, 106).

\section{Références bibliographiques}

Declercq, Gilles (1993). L'Art d'argumenter. Structures rhétoriques et littéraires, Paris, Editions universitaires.

Declercq, Gilles (1999). «La rhétorique classique entre évidence et sublime (1650-1675)», dans Histoire de la rhétorique dans l'Europe moderne, dans Histoire de la rhétorique dans l'Europe moderne (1450-1950), dir. Marc Fumaroli, Paris, PUF, 629-706.

Declercq, Gilles (2003). «L'identification des genres oratoires en tragédie française du XVII siècle (Iphigénie, Cinna) ». Theatrum mundi. Studies in Honor of Ronald W. Tobin, éd. Claire L. Carlin et Kathleen Wine, Charlottesville, Rookwood Press, 230-238.

Forestier, Georges (2006). Jean Racine, Paris, Gallimard.

Fumaroli, Marc (1980, 1994). L'Age de l'éloquence. Rhétorique et "res litteraria » de la Renaissance au seuil de l'époque classique [Genève, Droz], Paris, Albin Michel.

Lecompte, Jérôme (2007). Raison et vraisemblance à l'âge classique. Le statut de la rhétorique chez René Rapin et Jean Racine, thèse NR, dir. Gilles Declercq.

Molinié, Georges (1992). Dictionnaire de rhétorique, Librairie Générale Française.

Pernot, Laurent (2000). La Rhétorique dans l'Antiquité, Librairie Générale Française.

Quintilien (1975, 2003). Institution oratoire, éd. Jean Cousin, Paris, Les Belles Lettres.

Racine, Jean (1999). Euvres complètes, t. I, éd. Georges Forestier, Paris, Gallimard.

Rapin, René (1671). Réflexions sur l'usage de l'éloquence de ce temps, Paris, Claude Barbin, François Muguet.

Rapin, René $\left(1675^{2}, 1970\right)$. Réflexions sur la poétique de ce temps et sur les ouvrages des poètes anciens et modernes, éd. Elfrieda T. Dubois, Genève-Paris, Droz-Minard.

Saint-Évremond, Charles de (1681). «Fragment de Pétrone. De l'éloquence », dans CEuvres meslées, troisième partie, Paris, Claude Barbin, pp. 39-118.

Sénèque le Rhéteur (1932). Controverses et suasoires, éd. Henri Bornecque, Paris, Garnier.

Sénèque le Rhéteur (1992). Sentences, divisions et couleurs des orateurs et des rhéteurs, préface de Pascal Quignard, trad. revue par J.-H. Bornecque, Paris, Aubier.

1 . Le titre de la suasoire est le suivant: «Deliberat Agamemnon, an Iphigeniam immolet negante Calchante aliter navigari fas esse » («Agamemnon délibère s'il immolera Iphigénie, Calchas déclarant que, sans ce sacrifice, les dieux ne permettent pas de mettre à la voile »). Nous avons relevé l'existence de deux éditions incunables (1475 et 1492), suivies d'éditions procurées par Nicolas Le Fèvre, Andreas Schott, Juste-Lipse et Daniel Elzevier. Deux traductions françaises ont été plusieurs fois rééditées au cours du XVII ${ }^{\mathrm{e}}$ s., l'une par Matthieu Chalvet (de 1609 à 1643), l'autre par Bernard Lesfargues (de 1639 à 1669), ce qui témoigne de l'intérêt du siècle pour l'auteur. Au sujet des principales éditions, voir la bibliographie de Marc Fumaroli dans L'Age de l'éloquence, 733-734. Sur la 
distinction entre Sénèque père et fils et son importance au XVII ${ }^{\mathrm{e}}$ siècle, voir au même lieu, 172. Dans son récit intitulé Albucius, composé à partir de déclamations (P.O.L., 1990), Pascal Quignard indique avoir travaillé à partir d'une autre édition intéressante: Du Teil, Les Grandes déclamations de Quintilien, nouvellement traduites en françois, Paris, E. Loyson, 1658. Nous devons cette précision à G. Declercq, qui a donné des gages de sa lecture attentive de Quignard.

2 . Dans La Littérature européenne et le Moyen Age latin, Ernst R. Curtius évoque la continuité de la tradition déclamatoire, traitée au Moyen Age sous la forme de nouvelles, et note qu'un roman de Mlle de Scudéry, Ibrahim ou l'illustre Bassa (1641) est tiré d'une controverse de Sénèque l'Ancien, Controversia de archipiratae filia, I, 6 (Curtius, 259). Il convient d'ailleurs de souligner que la Clélie est à l'origine de la mode des Questions d'amour (voir César Rouben, «Un jeu de société au Grand Siècle: les Questions et les maximes d'amour. Inventaire chronologique ", Dix-septième siècle, 97, 1972), dont les Lettres portugaises sont l'expression littéraire la plus achevée. Les exemples donnés par Quintilien ont trouvé un écho à la Renaissance, en particulier dans une œuvre du Pogge, Un vieux doit-il se marier? (Paris, éd. V. Bruez, Les Belles Lettres, 1998), et plus encore avec le Tiers Livre de Rabelais (Panurge doit-il se marier ?). La Ratio studiorum des Jésuites a instauré pour les collèges des exercices de déclamation variés, dont la controverse, «sur le mode oratoire plutôt que dialectique », comme précisé au $\S 515$, où il est également traité de l'enjeu pédagogique de l'imitatio. Elle demeure la règle jusqu'aux alentours de 1660, après quoi la rhétorique entre en crise (François de Dainville, «L'évolution de l'enseignement de la rhétorique au XVII ${ }^{\mathrm{e}}$ siècle », dans L'Education des jésuites (XVI $-X V I I^{e}$ s.), Paris, Editions de Minuit, 1978, 185-208).

3 . Voir également De orat., II, XXXI, 133 sqq. Sur la pratique de Racine, voir sa critique des caractères d'Homère, dans Euvres, éd. R. Picard, Paris, Gallimard, 1966, t. II, 772, et G. Forestier, Jean Racine, Paris, Gallimard, 2006, 176-177.

${ }^{4}$. Voir L. Pernot, La Rhétorique dans l'Antiquité, 196.

5 . Saint-Évremond, «Fragment de Pétrone. De l'éloquence », dans Euvres mêlées, Paris, Claude Barbin, 1681, t. III. Nous résumons d'après cette traduction (voir Satiricon, II).

${ }^{6}$. Gabriel Guéret avait également averti de ce péril dans ses Entretiens sur l'éloquence de la chaire et du barreau (A Paris, chez Jean et René Guignard, 1666, 197-199).

${ }^{7}$. Georges Forestier signale pour ce vers un «point d'intonation»; la syntaxe suffisant à comprendre la phrase comme interrogative, il n'est pas utile de la ponctuer comme telle. Voir «Lire Racine », dans Euvres, éd. Gallimard, p. LXIII.

8 . Pour une étude de la dubitation, nous renvoyons à notre thèse, Troisième partie, chapitre II, «Sur des vers de Virgile et d'Ovide. Un lieu du pathétique : "Que faire ?" ", 449-479, à propos du monologue d'Hermione.

9 . Dans les éditions de 1687 puis 1697 , qui privilégient la lecture et non plus la déclamation, Racine remplacera ces deux points d'exclamation par des points d'interrogation.

${ }^{10}$. Voir Gilles Declercq (1999).

11 . Le texte des Remarques sur les Iphigénie de M. Racine et de M. Coras, figure dans l'édition des Euvres de Racine procurée par G. Forestier, 794-812.

12. Voir I, I, v. 67-68 et 83-88, puis IV, IV, v. 1221-1224.

13. Le mouvement des interrogations oratoires est repris de la préface de Racine (Euvres, 698).

14 . Voir Iphigénie, III, 6, v. 996-998. 